\title{
ABOUT THE EXISTENCE OF LOCALLY LIPSCHITZ OUTPUT FEEDBACK STABILIZERS FOR NONLINEAR SYSTEMS*
}

\author{
A. ISIDORI ${ }^{\dagger}$, L. PRALY ${ }^{\ddagger}$, AND L. MARCONI ${ }^{\S}$
}

\begin{abstract}
In this paper we complement some results of [L. Marconi, L. Praly, and A. Isidori, SIAM J. Control Optim., 45 (2007), pp. 2277-2298] by presenting a sufficient condition under which the output feedback controller proposed in that paper can be designed to be locally Lipschitz. The condition in question consists in a regularity property of the observable part of an autonomous system with output that generates the control input in a steady state. The work has been deliberately written to be an addendum to [L. Marconi, L. Praly, and A. Isidori, SIAM J. Control Optim., 45 (2007), pp. 2277-2298], to which the reader is referred for notation and main results.
\end{abstract}

Key words. output stabilization, nonlinear output regulation, locally Lipschitz regulators, nonlinear observers, robust control

AMS subject classifications. 93D15, 93D21, 93C10, 93B52, 93C15, 93D05

DOI. $10.1137 / 080741677$

1. Introduction. In this work we complement the results given in [10] by presenting a condition under which the regulator proposed in that paper is guaranteed to be locally Lipschitz. As in [10], we address a stabilization problem for the class of smooth nonlinear systems described by

$$
\begin{aligned}
& \dot{z}=f(z, y), \\
& \dot{y}=q(z, y)+b(z, y) u
\end{aligned}
$$

with state $(z, y) \in \mathbb{R}^{n} \times \mathbb{R}$, control input $u \in \mathbb{R}$, and measurable output $y$. The initial state $(z(0), y(0))$ is assumed to range in a known compact set $Z \times \Xi \subset \mathbb{R}^{n} \times \mathbb{R}$. The high frequency gain $b(z, y)$ is assumed to be bounded away from zero and, without loss of generality, positive. Associated with system (1) there is a controlled output $e \in \mathbb{R}^{p}$ defined as

$$
e=h(z, y)
$$

in which $h: \mathbb{R}^{n} \times \mathbb{R} \rightarrow \mathbb{R}^{p}$ is a smooth function. The "zero dynamics" (with respect to the input $u$ and output $y$ ) of system (1) are supposed to satisfy the following "minimum-phase" assumption.

Assumption $A$. There exists a compact set $\mathcal{A} \subset \mathbb{R}^{n}$ such that

$\left(\mathrm{a}_{1}\right)$ the set $\mathcal{A}$ is locally asymptotically stable for the system

$$
\dot{z}=f(z, 0)
$$

with a domain of attraction $\mathcal{D} \supset Z$; and

$\left(\mathrm{a}_{2}\right) h(z, 0)=0$ for all $z \in \mathcal{A}$.

*Received by the editors November 24, 2008; accepted for publication (in revised form) November 4, 2009; published electronically February 3, 2010.

http://www.siam.org/journals/sicon/48-5/74167.html

†DIS, Università di Roma "La Sapienza," 00184 Rome, Italy (isidori@ese.wustl.edu).

¥École des Mines de Paris, Fontainebleau, France (Laurent.Praly@mines-paristech.fr).

$\S$ C.A.SY. - DEIS, University of Bologna, 40136 Bologna, Italy (lorenzo.marconi@unibo.it). 
In this framework the control problem addressed in [10] was the one of designing an output feedback controller of the form

$$
\begin{aligned}
& \dot{\eta}=\varphi(\eta, y), \\
& u=\varrho(\eta, y)
\end{aligned}
$$

with state $\eta \in \mathbb{R}^{\nu}$ and a compact set $M \subset \mathbb{R}^{\nu}$ such that, in the associated closed-loop system

$$
\begin{aligned}
& \dot{z}=f(z, \zeta), \\
& \dot{\zeta}=q(z, \zeta)+\varrho(\eta, k(z, \zeta)), \\
& \dot{\eta}=\varphi(\eta, k(z, \zeta)), \\
& e=h(z, \zeta),
\end{aligned}
$$

the positive orbit of $Z \times \Xi \times M$ is bounded and, for each $(z(0), y(0), \eta(0)) \in Z \times \Xi \times M$,

$$
\lim _{t \rightarrow \infty} e(t)=0 .
$$

As shown in [10], this control problem encompasses stabilization problems by output feedback as well as problems of nonlinear output regulation. As far as the latter is concerned, a remarkable by-product of the results of [10] is a "universal" assumption-free technique for the design of the internal model and hence for the generation of the appropriate steady state control. This extends the domain of interest of the theory of nonlinear output regulation to meaningful previously untractable applications (see [8]).

The design solution proposed in [10] leads to a dynamical controller of the form

$$
\begin{aligned}
& \dot{\eta}=F \eta+G u, \quad \eta \in \mathbb{R}^{m}, \\
& u=\gamma(\eta)+v, \\
& v=\kappa(y),
\end{aligned}
$$

in which $(F, G) \in \mathbb{R}^{m \times m} \times \mathbb{R}^{m \times 1}$ is a controllable pair and $\gamma(\cdot)$ and $\kappa(\cdot)$ are properly designed continuous functions.

The design of $F, G, \gamma(\cdot)$, and $\kappa(\cdot)$ is achieved as follows. First, it is observed that the choice (5) yields a closed-loop system with relative degree 1 between the input $v$ and the output $y$ and zero dynamics of the form

$$
\begin{aligned}
& \dot{z}=f_{0}(z), \\
& \dot{\eta}=F \eta-G q_{0}(z),
\end{aligned}
$$

in which

$$
f_{0}(z):=f(z, 0), \quad q_{0}(z):=\frac{q(z, 0)}{b(z, 0)} .
$$

Proposition 1 of [10] shows that, if Assumption A holds and the matrix $F$ is Hurwitz, there exists a continuous function $\tau: \mathbb{R}^{n} \rightarrow \mathbb{R}^{m}$ such that the set

$$
\left.\operatorname{graph} \tau\right|_{\mathcal{A}}:=\left\{(z, \eta) \in \mathcal{A} \times \mathbb{R}^{m} \quad: \quad \eta=\tau(z)\right\}
$$

is locally asymptotically stable for (6) with a domain of attraction which contains $Z \times M$. Furthermore (see Proposition 2 in [10]), if the dimension $m$ of (5) satisfies $m \geq 2 n+2$, there is a number $\ell$ and a set $\mathcal{S} \in \mathbb{C}$ of zero Lebesgue measure such that, 
if the spectrum $\sigma(F)$ of $F$ satisfies $\sigma(F) \in\{\zeta \in \mathbb{C}: \operatorname{Re}(\zeta)<-\ell\} \backslash \mathcal{S}$, there always exists a class- $\mathcal{K}$ function $\rho(\cdot)$ for which the partial injectivity condition

$$
\left|q_{0}\left(z_{1}\right)-q_{0}\left(z_{2}\right)\right| \leq \rho\left(\left|\tau\left(z_{1}\right)-\tau\left(z_{2}\right)\right|\right) \quad \forall z_{1}, z_{2} \in \mathcal{A}
$$

holds. This, in turn, guarantees the existence of a continuous function $\gamma: \mathbb{R}^{m} \rightarrow \mathbb{R}$ such that (see Proposition 3 in [10])

$$
q_{0}(z)+\gamma \circ \tau(z)=0 \quad \forall z \in \mathcal{A} .
$$

In particular, if the function $\rho$ in (7) is linearly bounded at the origin, the function $\gamma$ can be chosen to be globally Lipschitz.

This being the case, it turns out that in the composite system

$$
\begin{aligned}
& \dot{z}=f(z, y), \\
& \dot{\eta}=F \eta+G[\gamma(\eta)+v], \\
& \dot{y}=q(z, y)+b(z, y)[\gamma(\eta)+v],
\end{aligned}
$$

the set graph $\left.\tau\right|_{\mathcal{A}} \times\{0\}$ is forward invariant when $v \equiv 0$, and, on this set, the controlled output $e$ is identically zero by assumption $\left(\mathrm{a}_{2}\right)$. Thus, to complete the design, it suffices to choose $v=\kappa(y)$ in such a way that the set graph $\left.\tau\right|_{\mathcal{A}} \times\{0\}$ is asymptotically stable, with a domain of attraction which contains the given set of initial conditions. As shown in Theorem 1 of [10], there always exists a continuous function $\kappa(\cdot)$ for which this is the case. In particular, the function $\kappa(\cdot)$ is linear if assumption $\left(\mathrm{a}_{1}\right)$ is strengthened by imposing that the set $\mathcal{A}$ be also locally exponentially stable for (2) and if the function $\gamma(\cdot)$ is locally Lipschitz (see Theorem 2 in [10]).

The results in [10] have been extended in subsequent works. In [7], several issues regarding the design of the stabilizer $v=\kappa(y)$ have been addressed by showing, in the context of nonlinear output regulation, a trade-off between the "regularity" of the stabilizer $v=\kappa(y)$ and the "minimality" of the internal model. In [8] possible exact and approximated expressions for the function $\gamma$ have been presented, proposing in this way a complete framework for exact and practical output regulation of nonlinear systems. In [3] the design technique in [10] has been applied to address some problems of output regulation for non-minimum-phase nonlinear systems. Then, in [11] and [9], the observer theory of [10] (and of [5]) has been applied in the context of nonlinear output feedback stabilization, under the additional hypothesis that the function $\gamma$ is locally Lipschitz.

An issue completely left open in [10] and in the subsequent works is the identification of conditions under which the function $\gamma$ (and thus the whole regulator (5)) is locally Lipschitz and not "only" continuous. This work is precisely meant to fill this gap. In the next section, we present a meaningful sufficient condition, expressed in terms of a checkable property of the functions $f_{0}$ and $q_{0}$, which guarantees that the function $\varrho$ in (7) is linearly bounded at the origin, and hence the regulator (5) is locally Lipschitz.

2. A sufficient condition for locally Lipschitz regulators. In what follows, we regard $f_{0}(\cdot)$ as a smooth vector field of $\mathbb{R}^{n}$ and let $\phi_{f_{0}}(t, z)$ denote its flow. With $f_{0}(\cdot)$ and the smooth function $q_{0}(\cdot)$ we associate a codistribution $\Omega$ defined as

$$
\Omega(z):=\sum_{k=0}^{\infty} \operatorname{span}\left\{\frac{\partial}{\partial z} L_{f_{0}}^{k} q_{0}(z)\right\}
$$


The next proposition provides a sufficient condition under which the regulator proposed in [10] is locally Lipschitz. The result is based upon two technical assumptions. The first is the following.

Assumption 1. There exists an open bounded subset $\mathcal{O} \supset \mathcal{A}$, where $\mathcal{A}$ is the compact set introduced in Assumption A, which is backward invariant for $\dot{z}=f_{0}(z)$.

The second technical assumption refers to the function $\tau: \mathcal{O} \rightarrow \mathbb{R}^{m}$ defined as

$$
\tau(z):=-\int_{-\infty}^{0} e^{-F s} G q_{0}\left(\phi_{f_{0}}(s, z)\right) d s,
$$

which, as shown in [10], is a function satisfying the properties (7) and (8). In Proposition 1 of [10] it was shown that the map in question is continuous and the set graph $\left.\tau\right|_{\mathcal{A}}$ is locally asymptotically stable for (6) with a domain of attraction containing $Z \times M$. Furthermore, if $m \geq 2 n+2$, there exist a set $\mathcal{S} \subset \mathbb{C}$ of zero Lebesgue measure and a positive $\ell$ such that if $\sigma(F) \in\{\zeta \in \mathbb{C}: \operatorname{Re}(\zeta)<\ell\} \backslash \mathcal{S}$, then there exist a class- $\mathcal{K}$ function $\varrho(\cdot)$ and a (at least) continuous function $\gamma: \mathbb{R}^{m} \rightarrow \mathbb{R}$ such that (7) and (8) hold (See Propositions 2 and 3 of [10]).

Assumption 2. The map $\tau: \mathcal{O} \rightarrow \tau(\mathcal{O})$ is open when $\tau(\mathcal{O})$ is equipped with the subset topology, i.e., for any $z^{\star} \in \mathcal{O}$ and for any $Z \subset \mathcal{O}$ open neighborhood of $z^{\star}$, there exists an open set $U \subset \mathbb{R}^{m}$, with $\tau\left(z^{\star}\right) \in U$, such that $U \cap \tau(\mathcal{O}) \subset \tau(Z)$.

We are now in a position to state the main result of the paper, which is the following proposition.

Proposition 2.1. Let Assumptions 1 and 2 hold. If there exists an integer $c$ such that

$$
\operatorname{dim}[\Omega(z)]=c \quad \forall z \in \mathcal{O},
$$

then there exists a globally Lipschitz function $\gamma(\cdot)$ satisfying (8).

The proof of this proposition relies on the following crucial lemma.

LEMMA 2.2. Under Assumption 1, if there exists an integer $c \leq n$ such that (11) holds, then, for any $z$ in $\mathcal{O}$, there exist a neighborhood $V_{z}$ and a real number $L_{z}$ such that we have

$$
\left|q_{0}\left(z_{1}\right)-q_{0}\left(z_{2}\right)\right| \leq L_{z}\left|\tau\left(z_{1}\right)-\tau\left(z_{2}\right)\right| \quad \forall z_{1}, z_{2} \in V_{z} .
$$

Proof of Lemma 2.2. Since $\mathcal{O}$ is bounded and backward invariant, $\phi_{f_{0}}(t, z)$ is defined for all $(t, z) \in(-\infty, 0) \times \mathcal{O}$ and gives rise to a smooth bounded function. Also, for each nonpositive $t, z \mapsto \phi_{f_{0}}(t, z)$ is a diffeomorphism. Set $y(t, z):=q_{0}\left(\phi_{f_{0}}(t, z)\right)$. For each $z$, the function $t \in(-\infty, 0] \mapsto y(t, z)$ is bounded. Also, for each $t$ in $(-\infty, 0]$ and any $k$ in $\mathbb{N}$, we have

$$
\frac{\mathrm{d}^{k} y(t, z)}{\mathrm{d} t^{k}}=L_{f_{0}}^{k} q_{0}\left(\phi_{f_{0}}(t, z)\right) .
$$

Let $(F, G) \in \mathbb{R}^{m \times m} \times \mathbb{R}^{m \times 1}$ be a controllable pair with $F$ Hurwitz, and define $\tau: \mathcal{O} \rightarrow \mathbb{R}^{m}$ as in (10). This map is continuous and satisfies

$$
L_{f_{0}} \tau(z)=F \tau(z)-G q_{0}(z) \quad \forall z \in \mathcal{O} .
$$

Observe that

$$
\frac{\partial \phi_{f_{0}}}{\partial z}(0, z)=I, \quad \frac{\partial^{2} \phi_{f_{0}}}{\partial z^{2}}(0, z)=0
$$


and that

$$
\frac{\partial^{2} \phi_{f_{0}}}{\partial s \partial z}(s, z)=\frac{\partial f_{0}}{\partial z}\left(\phi_{f_{0}}(s, z)\right) \frac{\partial \phi_{f_{0}}}{\partial z}(s, z)
$$

and

$$
\begin{aligned}
\frac{\partial^{3} \phi_{f_{0}}}{\partial s \partial z^{2}}(s, z)= & \frac{\partial f_{0}}{\partial z}\left(\phi_{f_{0}}(s, z)\right) \frac{\partial^{2} \phi_{f_{0}}}{\partial z^{2}}(s, z) \\
& +\left(\frac{\partial^{2} f_{0}}{\partial z^{2}}\left(\phi_{f_{0}}(s, z)\right) \times \frac{\partial \phi_{f_{0}}}{\partial z}(s, z)\right) \frac{\partial \phi_{f_{0}}}{\partial z}(s, z) .
\end{aligned}
$$

Also, if $z$ is in $\mathcal{O}$, then $\phi_{f_{0}}(s, z)$ is in the compact set $\mathrm{Cl}(\mathcal{O})$ for all $s \leq 0$. By integrating (14) with the initial condition given by the first equation in (13), it follows that $\left|\frac{\partial \phi_{f_{0}}}{\partial z}(s, z)\right|$ does not grow in $s$ faster than $e^{F_{1}|s|}$, where $F_{1}$ is a bound of $\left|\frac{\partial f_{0}}{\partial z}\right|$ on $\mathrm{Cl}(\mathcal{O})$. Furthermore, by integrating (15) with the initial condition given by the second equation of (13) and by taking into account the previous bound, it follows that $\left|\frac{\partial^{2} \phi_{f_{0}}}{\partial z^{2}}(s, z)\right|$ does not grow in $s$ faster than $e^{2 F_{1}|s|}$. So, when the eigenvalues of $F$ have real part strictly smaller than $\ell=-2 F_{1}$, the function $\tau$ is $C^{2}$ on $\mathcal{O}$.

Finally, observe that

$$
\frac{\partial \tau}{\partial z}(z)=-\int_{-\infty}^{0} e^{-F s} G \frac{\partial y}{\partial z}(s, z) d s
$$

In the remaining part of the proof we prove that, under the condition expressed in Lemma 2.2, condition (12) holds. For clarity, we have divided the overall proof into a number of claims.

Claim 1. For any $z \in \mathcal{O}$, any ${ }^{1} v \in \mathbb{R}^{n} \backslash \Omega^{\perp}(z)$, and any $T<0$, there exists $t_{-}(z, v, T)<t_{+}(z, v, T)$ in $[T, 0]$ satisfying

$$
\frac{\partial y}{\partial z}(t, z) \cdot v \neq 0 \quad \forall t \in\left(t_{-}(z, v, T), t_{+}(z, v, T)\right) .
$$

Proof. If this were not the case, by continuity in $t$, there would exist $z$ in $\mathcal{O}, v$ in $\mathbb{R}^{n} \backslash \Omega^{\perp}(z)$, and $T<0$ satisfying

$$
\frac{\partial y}{\partial z}(t, z) \cdot v=0 \quad \forall t \in[T, 0]
$$

and therefore

$$
0=\frac{\mathrm{d}^{k}}{\mathrm{~d} t^{k}} \frac{\partial y}{\partial z}(t, z) \cdot v=\frac{\partial L_{f_{0}}^{k} q_{0}}{\partial z}\left(\phi_{f_{0}}(t, z)\right) \frac{\partial \phi_{f_{0}}}{\partial z}(t, z) \cdot v \quad \forall t \in(T, 0), \forall k \in \mathbb{N} .
$$

Hence by continuity at 0 in $t$ and since we have

$$
\phi_{f_{0}}(0, z)=z, \quad \frac{\partial \phi_{f_{0}}}{\partial z}(0, z)=I,
$$

by taking the limit as $t \rightarrow 0^{-}$, we would obtain

$$
0=\frac{\partial L_{f_{0}}^{k} q_{0}}{\partial z}(z) \cdot v \quad \forall k \in \mathbb{N}
$$

\footnotetext{
${ }^{1}$ We denote by $\Omega^{\perp}(z)$ the annihilator of $\Omega(z)$ defined as $\Omega^{\perp}(z)=\left\{w \in\left(\mathbb{R}^{n}\right)^{\star}:\langle w, v\rangle=\right.$ 0 for all $v \in \Omega(z)\}$, where $\left(\mathbb{R}^{n}\right)^{\star}$ is the dual space of $\mathbb{R}^{n}$.
} 
This says that $v$ would be in $\Omega^{\perp}(z)$, which is a contradiction.

Claim 2. For any $z \in \mathcal{O}$ and any $v \in \Omega^{\perp}(z)$, we have

$$
\frac{\partial y}{\partial z}(t, z) \cdot v=0 \quad \forall t \leq 0 .
$$

Proof. Pick arbitrary $z$ in $\mathcal{O}$. Since $y(t, z)=q_{0}\left(\phi_{f_{0}}(t, z)\right)$, we have

$$
\frac{\partial y}{\partial z}(t, z)=\frac{\partial q_{0}}{\partial z}\left(\phi_{f_{0}}(t, z)\right) \frac{\partial \phi_{f_{0}}}{\partial z}(t, z) .
$$

But following [6, Lemma 3.6], we know that condition (11) implies

$$
\Omega\left(\phi_{f_{0}}(t, z)\right) \frac{\partial \phi_{f_{0}}}{\partial z}(t, z)=\Omega(z) \quad \forall t \leq 0, \forall z \in \mathcal{O},
$$

or, similarly,

$$
\frac{\partial \phi_{f_{0}}}{\partial z}(t, z) \Omega^{\perp}(z)=\Omega^{\perp}\left(\phi_{f_{0}}(t, z)\right) .
$$

Hence, for any $v$ in $\Omega^{\perp}(z), \frac{\partial \phi_{f_{0}}}{\partial z}(t, z) v$ is a vector in $\Omega^{\perp}\left(\phi_{f_{0}}(t, z)\right)$, which therefore annihilates $\frac{\partial q_{0}}{\partial z}\left(\phi_{f_{0}}(t, z)\right)$.

In the following claim we refer to the integer $c$ introduced in (11).

Claim 3. For any $z_{j} \in \mathcal{O}$, there exist a connected neighborhood $U_{j}$ of $z_{j}$ and a diffeomorphism $\Phi_{j}: U_{j} \rightarrow \mathbb{R}^{n}$ such that, by choosing the coordinates $x=\Phi_{j}(z)$ defined on $U_{j}$, for any $x$ in $\Phi_{j}\left(U_{j}\right)$ we have the following:

1. For any $v \notin \Omega^{\perp}\left(\Phi_{j}^{-1}(x)\right)$, there exist $w_{a} \in \mathbb{R}^{c} \backslash\{0\}$ and $v_{0} \in \Omega^{\perp}\left(\Phi_{j}^{-1}(x)\right)$ satisfying

$$
v=\Psi_{a, j}(x) w_{a}+v_{0},
$$

where $\Psi_{a, j}(x)$ is the left invertible matrix given by the first $c$ columns of $\frac{\partial \Phi_{j}^{-1}}{\partial x}(x)$ and, conversely, for any $w_{a} \in \mathbb{R}^{c} \backslash\{0\}$ and $v_{0} \in \Omega^{\perp}\left(\Phi_{j}^{-1}(x)\right)$, the vector (18) satisfies $v \notin \Omega^{\perp}\left(\Phi_{j}^{-1}(x)\right)$.

2. For any $v \in \Omega^{\perp}\left(\Phi_{j}^{-1}(x)\right)$, there exists $w_{b}$ in $\mathbb{R}^{n-c}$ satisfying

$$
v=\Psi_{b, j}(x) w_{b},
$$

where $\Psi_{b, j}(x)$ is the left invertible matrix given by the $n-c$ last columns of $\frac{\partial \Phi_{j}^{-1}}{\partial x}(x)$ and, conversely, for any $w_{b}$ in $\mathbb{R}^{n-c}$, the vector (19) satisfies $v \in \Omega^{\perp}\left(\Phi_{j}^{-1}(x)\right)$.

Proof. Since $\Omega(z)$ has dimension $c$ at $z_{j}$, there exist integers $k_{1}, \ldots, k_{c}$ such that

$$
\Omega\left(z_{j}\right)=\sum_{i=1}^{c} \operatorname{span}\left\{\frac{\partial L_{f_{0}}^{k_{i}} q_{0}}{\partial z}\left(z_{j}\right)\right\} .
$$

Set

$$
\varphi_{i}(z)=L_{f_{0}}^{k_{i}} q_{0}(z), \quad i=1, \ldots, c .
$$

By continuity and condition (11), there exists a neighborhood $W_{j}$ of $z_{j}$ such that the $c$ covectors

$$
\frac{\partial \varphi_{1}}{\partial z}(z), \ldots, \frac{\partial \varphi_{c}}{\partial z}(z)
$$


are linearly independent at each $z \in W_{j}$ and $\operatorname{span} \Omega(z)$, i.e.,

$$
\Omega(z)=\sum_{i=1}^{c} \operatorname{span}\left\{\frac{\partial \varphi_{i}}{\partial z}(z)\right\}, \quad z \in W_{j}
$$

As a consequence, for each $k$ in $\mathbb{N}$, there exists a set of $c$ continuous functions $c_{k i}$, defined on $W_{j}$, satisfying

$$
\frac{\partial L_{f_{0}}^{k} q_{0}}{\partial z}(z)=\sum_{i=1}^{r} c_{k i}(z) \frac{\partial \varphi_{i}}{\partial z}(z) \quad \forall z \in W_{j} .
$$

Since the covectors in (20) are linearly independent at $z_{j}$, it is always possible to find a set of additional $n-c$ smooth functions

$$
\varphi_{c+1}(z), \ldots, \varphi_{n}(z)
$$

such that the $n$ covectors

$$
\frac{\partial \varphi_{1}}{\partial z}(z), \ldots, \frac{\partial \varphi_{n}}{\partial z}(z)
$$

are linearly independent at $z_{j}$. As a consequence, there exists a neighborhood $U_{j}$ of $z_{j}$ such that the function $\Phi_{j}: U_{j} \rightarrow \mathbb{R}^{n}$ defined as

$$
\Phi_{j}(z):=\operatorname{col}\left\{\varphi_{1}(z), \ldots, \varphi_{n}(z)\right\}
$$

is a diffeomorphism. Without loss of generality, we may assume $U_{j}$ is a subset of $W_{j}$ and is connected.

Let the $n$ vectors $v_{1}, \ldots, v_{n}$ be defined as

$$
v_{i}=\frac{\partial \Phi_{j}^{-1}}{\partial x}(x) e_{i},
$$

where $e_{i}$ is the $i$ th standard basis vector in $\mathbb{R}^{n}$. They are also the $n$ columns of $\frac{\partial \Phi_{j}^{-1}}{\partial x}(x)$ which we have decomposed as

$$
\left[v_{1}, \ldots, v_{c}\right]=\Psi_{a, j}(x), \quad\left[v_{c+1}, \ldots, v_{n}\right]=\Psi_{b, j}(x),
$$

where $\Psi_{a, j}(x)$ and $\Psi_{b, j}(x)$ are, respectively, the first $c$ and $n-c$ last columns of $\frac{\partial \Phi_{j}^{-1}}{\partial x}(x)$. Each $v_{i}$ can be viewed as a tangent vector to the $n$-dimensional manifold $U_{j}$ at the point $z=\Phi_{j}^{-1}(x)$. And, since we have

$$
\frac{\partial \Phi_{j}}{\partial z}\left(\Phi_{j}^{-1}(x)\right) \frac{\partial \Phi_{j}^{-1}}{\partial x}(x)=I
$$

we obtain

$$
\frac{\partial \Phi_{j}}{\partial z}\left(\Phi_{j}^{-1}(x)\right)\left[v_{1} \cdots v_{n}\right]=I \quad \forall x \in \Phi_{j}\left(U_{j}\right) .
$$

Bearing in mind the definition of $\Phi_{j}$, this is equivalent to

$$
\frac{\partial \varphi_{i}}{\partial z}(z) v_{k}=\delta_{i k} \quad \forall z \in U_{j}
$$


where $\delta_{i k}$ is the Kroeneker symbol. In particular, we obtain that if $i \leq c$ and $k \geq c+1$, then

$$
\frac{\partial \varphi_{i}}{\partial z}(z) v_{k}=0 \quad \forall z \in U_{j} .
$$

Because of (21), this implies

$$
v_{k} \in \Omega^{\perp}(z) \quad \forall k \geq c+1, \quad \forall z \in U_{j} .
$$

Since these $n-c$ vectors are linearly independent, a dimensionality argument allows us to conclude that

$$
\Omega^{\perp}(z)=\operatorname{span}\left\{v_{c+1}, \ldots, v_{n}\right\} \quad \forall z \in U_{j} .
$$

This implies the second point of the statement.

For the same reason, we see that any nontrivial linear combination of $v_{1}, \ldots, v_{c}$ is not in $\Omega^{\perp}(z)$ (otherwise, there would be a point $z$ in $U_{j}$ at which $\Omega^{\perp}(z)$ would have dimension higher than $n-c$, thus contradicting the basic hypothesis).

Now, pick any $z$ in $U_{j}$ and any $v \notin \Omega^{\perp}(z)$, that is, any $x$ in $\Phi_{j}\left(U_{j}\right)$ and any $v \notin \Omega^{\perp}\left(\Phi_{j}^{-1}(x)\right)$. The vector $v$ has necessarily nonzero components along $v_{1}, \ldots, v_{c}$. Therefore, there exist $w_{a}$ in $\mathbb{R}^{c} \backslash\{0\}$ and $v_{0}$ in $\Omega^{\perp}\left(\Phi_{j}^{-1}(x)\right)$ such that

$$
v=\Psi_{a, j}(x) w_{a}+v_{0},
$$

which concludes the proof of the claim.

To simplify the following notation, we let $\Upsilon_{j}$ be the open set

$$
\Upsilon_{j}=\Phi_{j}\left(U_{j}\right) \times \mathbb{R}^{c} \backslash\{0\} \subset \mathbb{R}^{n+c} .
$$

Note that, $U_{j}$ being open, for each $z \in U_{j}$ there exists $T(j, z)<0$ such that $\phi_{f_{0}}(t, z)$ is in $U_{j}$ for all $t$ in $[T(j, z), 0]$. We have established the following facts:

1. From Claims 1 and 3 , for each pair $\left(x, w_{a}\right)$ in $\Upsilon_{j}$, there exist $t_{-}<t_{+}$defined as $t_{-}=t_{-}\left(\Phi_{j}^{-1}(x), \Psi_{a, j}(x) w_{a}, T\left(j, \Phi_{j}^{-1}(x)\right)\right)$ and $t_{+}=t_{+}\left(\Phi_{j}^{-1}(x), \Psi_{a, j}(x) w_{a}\right.$, $\left.T\left(j, \Phi_{j}^{-1}(x)\right)\right)$ and satisfying

$$
\frac{\partial y}{\partial z}\left(t, \Phi_{j}^{-1}(x)\right) \cdot \Psi_{a, j}(x) w_{a} \neq 0 \quad \forall t \in\left(t_{-}, t_{+}\right) .
$$

2. From Claims 2 and 3 , for each pair $\left(x, w_{b}\right)$ in $\Phi_{j}\left(U_{j}\right) \times \mathbb{R}^{n-c}$, we have

$$
\frac{\partial y}{\partial z}\left(t, \Phi_{j}^{-1}(x)\right) \cdot \Psi_{b, j}(x) w_{b}=0 \quad \forall t \leq 0 .
$$

Motivated by the previous results, let us split the coordinates $x$ into two parts $x_{a}$, collecting the first $c$ coordinates, and $x_{b}$, collecting the $n-c$ last ones, and define, for each $x$ in $\Phi_{j}\left(U_{i}\right)$,

$$
\bar{q}_{0 j}\left(x_{a}, x_{b}\right)=q_{0}\left(\Phi_{j}^{-1}(x)\right), \quad \bar{y}_{j}\left(t, x_{a}, x_{b}\right)=y\left(t, \Phi_{j}^{-1}(x)\right) .
$$

With this notation, points 1 and 2 above yield, respectively, the following.

1. For each triplet $\left(\left(x_{a}, x_{b}\right), w_{a}\right)$ in $\Upsilon_{j}$, there exist $t_{-}<t_{+}$satisfying

$$
\frac{\partial \bar{y}_{j}}{\partial x_{a}}\left(t, x_{a}, x_{b}\right) w_{a} \neq 0 \quad \forall t \in\left(t_{-}, t_{+}\right) .
$$


2. For each pair $\left(x_{a}, x_{b}\right)$ in $\Phi_{j}\left(U_{j}\right)$, we have

$$
\frac{\partial \bar{y}_{j}}{\partial x_{b}}\left(t, x_{a}, x_{b}\right)=0 \quad \forall t \leq 0 .
$$

From now on, we adapt to the present context arguments which have been used in [1]. Let $\Lambda$ be the set $\{\lambda \in \mathbb{C}: \operatorname{Re}(\lambda)<\ell\}$. From the fact that $\tau$ is $C^{2}$ on $\mathcal{O}$, it follows also that the function $g: \mathcal{O} \times \Lambda \rightarrow \mathbb{C}^{n}$ defined as

$$
g(z, \lambda)=\int_{-\infty}^{0} e^{-\lambda s} \frac{\partial y}{\partial z}(s, z) d s
$$

is $C^{1}$ in $z$ for each $\lambda$ and holomorphic in $\lambda$ for each $z$. Then, by the definition (24), the function $g_{a, j}: \Phi_{j}\left(U_{j}\right) \times \Lambda \rightarrow \mathbb{C}^{c}$ defined as

$$
\begin{aligned}
\bar{g}_{a, j}\left(x_{a}, x_{b}, \lambda\right) & =g\left(\Phi_{j}^{-1}\left(\left(x_{a}, x_{b}\right)\right), \lambda\right) \Psi_{a, j}\left(\left(x_{a}, x_{b}\right)\right) \\
& =\int_{-\infty}^{0} e^{-\lambda s} \frac{\partial \bar{y}_{j}}{\partial x_{a}}\left(s, x_{a}, x_{b}\right) d s
\end{aligned}
$$

is $C^{1}$ in $\left(x_{a}, x_{b}\right)$ for each $\lambda$ and holomorphic in $\lambda$ for each $\left(x_{a}, x_{b}\right)$.

We recall now a lemma proved in [2].

Lemma 2.3. Let $\Lambda$ and $\Upsilon$ be open subsets of $\mathbb{C}$ and $\mathbb{R}^{n+c}$, respectively. Let $(\chi, \lambda) \mapsto v(\chi, \lambda)$ be a complex valued function defined on $\Upsilon \times \Lambda$ which is holomorphic in $\lambda$ for each $\chi \in \Upsilon$ and $C^{1}$ for each $\lambda \in \Lambda$. If, for each pair $(\chi, \lambda)$ in $\Upsilon \times \Lambda$ for which $v(\chi, \lambda)$ is zero, we can find an integer $k$ satisfying

$$
\left\{\begin{array}{l}
\frac{\partial^{i} v}{\partial \lambda^{i}}(\chi, \lambda)=0 \quad \forall i \in\{0, \ldots, k-1\} \\
\frac{\partial^{k} v}{\partial \lambda^{k}}(\chi, \lambda) \neq 0
\end{array}\right.
$$

then the set

$$
S=\bigcup_{\chi \in \Upsilon}\left\{\left(\lambda_{1}, \ldots, \lambda_{n+1}\right) \in \Lambda^{n+c+1}: v\left(\chi, \lambda_{1}\right)=\cdots=v\left(\chi, \lambda_{n+c+1}\right)=0\right\}
$$

has zero Lebesgue measure in $\mathbb{C}^{n+c+1}$.

In our context the previous lemma is used with $\chi$ defined as the triplet $\left(x_{a}, x_{b}, w_{a}\right)$ in the open set $\Upsilon_{j}$ and the function $v$ as

$$
v(\chi, \lambda)=\bar{g}_{j}\left(x_{a}, x_{b}, \lambda\right) w_{a} .
$$

Observe that (22) implies

$$
\int_{-\infty}^{0} e^{-2 \operatorname{Re}(\lambda) s}\left|\frac{\partial \bar{y}_{j}}{\partial x_{a}}\left(s, x_{a}, x_{b}\right) w_{a}\right|^{2} d s>0 \quad \forall\left(\left(x_{a}, x_{b}, w_{a}\right), \lambda\right) \in \Upsilon_{j} \times \Lambda .
$$

So, from Parseval's theorem, condition (25) of Lemma 2.3 is satisfied. It follows that, if $\left(\lambda_{i}\right)$ is a set of $(n+c+1)$ complex numbers chosen in $\Lambda^{n+c+1} \backslash S_{j}$, where $S_{j}$, associated to $\Upsilon_{j}$, is the set given by Lemma 2.3, we have

$$
\left(\begin{array}{c}
\bar{g}_{j}\left(x_{a}, x_{b}, \lambda_{1}\right) \\
\vdots \\
\bar{g}_{j}\left(x_{a}, x_{b}, \lambda_{n+c+1}\right)
\end{array}\right) w_{a} \neq 0 \quad \forall\left(x_{a}, x_{b}, w_{a}\right) \in \Upsilon_{j}
$$


But, $\mathcal{O}$ being locally compact, there exists an open set $V_{j}$ containing $z_{j}$ whose closure $\mathrm{Cl}\left(V_{j}\right)$ is compact and contained in $U_{j}$. And, $\mathcal{O}$ being paracompact, there exists a countable set $J$ of $z_{j}$ 's satisfying

$$
\mathcal{O} \subset \bigcup_{i \in J} V_{j}
$$

Then since $S_{j}$ is of zero Lebesgue measure and $J$ is countable, the set

$$
S=\bigcup_{j \in J} S_{j}
$$

has zero Lebesgue measure, and, by picking $\left(\lambda_{i}\right)$ in $\Lambda^{n+c+1} \backslash S$, we have

$$
\left(\begin{array}{c}
\bar{g}_{j}\left(x_{a}, x_{b}, \lambda_{1}\right) \\
\vdots \\
\bar{g}_{j}\left(x_{a}, x_{b}, \lambda_{n+c+1}\right)
\end{array}\right) w_{a} \neq 0 \quad \forall\left(x_{a}, x_{b}, w_{a}\right) \in \Upsilon_{j}, \forall j \in J
$$

By choosing the pair $(F, G)$ as

$$
F=\operatorname{diag}\left(\lambda_{1}, \ldots, \lambda_{n+c+1}\right), \quad G=\left(\begin{array}{lll}
1 & \ldots & 1
\end{array}\right)^{T},
$$

by defining the function $\bar{\tau}_{j}$ as

$$
\bar{\tau}_{j}\left(x_{a}, x_{b}\right)=\tau\left(\Phi_{j}^{-1}\left(x_{a}, x_{b}\right)\right)=-\int_{-\infty}^{0} e^{-F s} G \bar{y}_{j}\left(s, x_{a}, x_{b}\right) d s
$$

and by comparing (24) and (16), it follows that

$$
\frac{\partial \bar{\tau}_{j}}{\partial x_{a}}\left(x_{a}, x_{b}\right) w_{a}=-\left(\begin{array}{c}
\bar{g}_{j}\left(x_{a}, x_{b}, \lambda_{1}\right) \\
\vdots \\
\bar{g}_{j}\left(x_{a}, x_{b}, \lambda_{n+c+1}\right)
\end{array}\right) w_{a} \neq 0 \quad \forall\left(x_{a}, x_{b}, w_{a}\right) \in \Upsilon_{j}, \forall j \in J
$$

This implies

$$
\operatorname{det}\left(\left(\frac{\partial \bar{\tau}_{j}}{\partial x_{a}}\left(x_{a}, x_{b}\right)\right)^{T}\left(\frac{\partial \bar{\tau}_{j}}{\partial x_{a}}\left(x_{a}, x_{b}\right)\right)\right) \neq 0 \quad \forall\left(x_{a}, x_{b}\right) \in \Phi_{j}\left(U_{j}\right), \forall j \in J .
$$

Hence, for each $j$ in $J$, the functions $M_{j}: \Phi_{j}\left(U_{j}\right) \rightarrow \mathbb{R}^{c \times 2(n+c+1)}$ and $N_{j}: \Phi_{j}\left(U_{j}\right) \rightarrow$ $\mathbb{R}^{1 \times 2(n+c+1)}$, defined as

$$
\begin{aligned}
M_{j}\left(x_{a}, x_{b}\right) & =\left(\frac{\partial \bar{\tau}_{j}}{\partial x_{a}}\left(x_{a}, x_{b}\right)^{T} \frac{\partial \bar{\tau}_{j}}{\partial x_{a}}\left(x_{a}, x_{b}\right)\right)^{-1} \frac{\partial \bar{\tau}_{j}}{\partial x_{a}}\left(x_{a}, x_{b}\right)^{T} \\
N_{j}\left(x_{a}, x_{b}\right) & =\frac{\partial \bar{q}_{0 j}}{\partial x_{a}}\left(x_{a}, x_{b}\right) M_{j}\left(x_{a}, x_{b}\right)
\end{aligned}
$$

are continuous and satisfy, for all $\left(x_{a}, x_{b}\right)$ in $\Phi_{j}\left(U_{j}\right)$,

$$
M_{j}\left(x_{a}, x_{b}\right) \frac{\partial \bar{\tau}_{j}}{\partial x_{a}}\left(x_{a}, x_{b}\right)=I
$$


and

$$
N_{j}\left(x_{a}, x_{b}\right) \frac{\partial \bar{\tau}_{j}}{\partial x_{a}}\left(x_{a}, x_{b}\right)=\frac{\partial \bar{q}_{0 j}}{\partial x_{a}}\left(x_{a}, x_{b}\right) .
$$

On the other hand, (23) gives

$$
\frac{\partial \bar{\tau}_{j}}{\partial x_{b}}\left(x_{a}, x_{b}\right)=0, \quad \frac{\partial \bar{q}_{0 j}}{\partial x_{b}}\left(x_{a}, x_{b}\right)=0 \quad \forall\left(x_{a}, x_{b}\right) \in \Phi_{j}\left(U_{j}\right), \forall j \in J .
$$

Since $\Phi_{j}\left(U_{j}\right)$ is connected, this implies that $\bar{\tau}_{j}$ and $\bar{q}_{0 j}$ do not depend on $x_{b}$.

Now for any $z_{*}$ in $\mathcal{O}$, there exist $j$ in $J$ such that $V_{j}$ contains $z_{*}$. Let $\left(z_{1}, z_{2}\right)$ be an arbitrary pair in $\mathrm{Cl}\left(V_{j}\right)^{2}$. There exist two points $x_{1}=\left(x_{a, 1}, x_{b, 1}\right)$ and $x_{2}=\left(x_{a, 2}, x_{b, 2}\right)$ in $\Phi_{j}\left(\mathrm{Cl}\left(V_{j}\right)\right) \subset U_{j}^{2}$ satisfying

$$
\begin{aligned}
& z_{1}=\Phi_{j}^{-1}\left(\left(x_{a, 1}, x_{b, 1}\right)\right), \quad z_{2}=\Phi_{j}^{-1}\left(\left(x_{a, 2}, x_{b, 2}\right)\right), \\
& \tau\left(z_{1}\right)=\bar{\tau}_{j}\left(x_{a, 1}, x_{b, 1}\right), \quad \tau\left(z_{2}\right)=\bar{\tau}_{j}\left(x_{a, 2}, x_{b, 2}\right),
\end{aligned}
$$

and

$$
q_{0}\left(z_{1}\right)=\bar{q}_{0 j}\left(x_{a, 1}, x_{b, 1}\right), \quad q_{0}\left(z_{2}\right)=\bar{q}_{0 j}\left(x_{a, 2}, x_{b, 2}\right) .
$$

Let $\Delta_{\tau}$ and $\Delta_{q_{0}}$ be defined on $\mathrm{Cl}\left(V_{j}\right)^{2}$ as

$$
\begin{gathered}
\Delta_{\tau}\left(z_{1}, z_{2}\right)=\tau\left(z_{1}\right)-\tau\left(z_{2}\right)-\left(\frac{\partial \tau}{\partial z}\left(z_{1}\right) \frac{\partial \Phi_{j}^{-1}}{\partial x}\left(x_{1}\right)\right)\left(x_{1}-x_{2}\right), \\
\Delta_{q_{0}}\left(z_{1}, z_{2}\right)=q_{0}\left(z_{1}\right)-q_{0}\left(z_{2}\right)-\left(\frac{\partial q_{0}}{\partial z}\left(z_{1}\right) \frac{\partial \Phi_{j}^{-1}}{\partial x}\left(x_{1}\right)\right)\left(x_{1}-x_{2}\right) .
\end{gathered}
$$

Since $\bar{\tau}_{j}$ and $\bar{q}_{0 j}$ do not depend on $x_{b}$, we have

$$
\begin{aligned}
\Delta_{\tau}\left(z_{1}, z_{2}\right) & =\bar{\tau}_{j}\left(x_{a, 1}, x_{b, 1}\right)-\bar{\tau}_{j}\left(x_{a, 2}, x_{b, 1}\right)-\frac{\partial \bar{\tau}_{j}}{\partial x_{a}}\left(x_{a, 1}, x_{b, 1}\right)\left(x_{a, 1}-x_{a, 2}\right), \\
\Delta_{q_{0}}\left(z_{1}, z_{2}\right) & =\bar{q}_{0 j}\left(x_{a, 1}, x_{b, 1}\right)-\bar{q}_{0 j}\left(x_{a, 2}, x_{b, 1}\right)-\frac{\partial \bar{q}_{0 j}}{\partial x_{a}}\left(x_{a, 1}, x_{b, 1}\right)\left(x_{a, 1}-x_{a, 2}\right) .
\end{aligned}
$$

Hence, $\bar{\tau}_{j}$ and $\bar{q}_{0 j}$ being $C^{2}$, there exists $\delta>0$ such that we have

$$
\frac{\left|\Delta_{\tau}\left(z_{1}, z_{2}\right)\right|}{\left|x_{a, 1}-x_{a, 2}\right|} \leq \frac{1}{2 M_{j, \max }}, \frac{\left|\Delta_{q_{0}}\left(z_{1}, z_{2}\right)\right|}{\left|x_{a, 1}-x_{a, 2}\right|} \leq \frac{N_{j, \max }}{2 M_{j, \max }} \quad \forall\left(z_{1}, z_{2}\right) \in V_{j}^{2}:\left|z_{1}-z_{2}\right| \leq \delta,
$$

where $M_{j, \max }$ and $N_{j, \max }$ denote an upperbound of $\left|M_{j}\right|$ and $\left|N_{j}\right|$ on the compact set $\Phi_{j}\left(\mathrm{Cl}\left(V_{j}\right)\right)$. Then, setting

$$
x_{a, 1}-x_{a, 2}=M_{j}\left(x_{a, 1}, x_{b, 1}\right)\left[\Delta_{\tau}\left(z_{1}, z_{2}\right)-\left(\tau\left(z_{1}\right)-\tau\left(z_{2}\right)\right)\right]
$$

yields

$$
\left|x_{a, 1}-x_{a, 2}\right| \leq M_{j, \max }\left|\tau\left(z_{1}\right)-\tau\left(z_{2}\right)\right|+\frac{1}{2}\left|x_{a, 1}-x_{a, 2}\right| \quad \forall\left(z_{1}, z_{2}\right) \in V_{j}^{2}:\left|z_{1}-z_{2}\right| \leq \delta .
$$


With all this, we get

$$
\begin{aligned}
\left|q_{0}\left(z_{1}\right)-q_{0}\left(z_{2}\right)\right| & =\left|\left(\frac{\partial q_{0}}{\partial z}\left(z_{1}\right) \frac{\partial \Phi_{j}^{-1}}{\partial x}\left(x_{1}\right)\right)\left(x_{1}-x_{2}\right)+\Delta_{q_{0}}\left(z_{1}, z_{2}\right)\right| \\
& =\left|\frac{\partial \bar{q}_{0 j}}{\partial x_{a}}\left(x_{a, 1}, x_{b, 1}\right)\left(x_{a, 1}-x_{a, 2}\right)+\Delta_{q_{0}}\left(z_{1}, z_{2}\right)\right| \\
& =\left|N_{j}\left(x_{a, 1}, x_{b, 1}\right) \frac{\partial \bar{\tau}_{j}}{\partial x_{a}}\left(x_{a, 1}, x_{b, 1}\right)\left(x_{a, 1}-x_{a, 2}\right)+\Delta_{q_{0}}\left(z_{1}, z_{2}\right)\right| \\
& =\left|N_{j}\left(x_{a, 1}, x_{b, 1}\right)\left[\tau\left(z_{1}\right)-\tau\left(z_{2}\right)-\Delta_{\tau}\left(z_{1}, z_{2}\right)\right]+\Delta_{q_{0}}\left(z_{1}, z_{2}\right)\right| \\
& \leq N_{j, \max }\left|\tau\left(z_{1}\right)-\tau\left(z_{2}\right)\right|+\frac{N_{j, \max }}{M_{j, \max }}\left|x_{a, 1}-x_{a, 2}\right| \\
& \leq 3 N_{j, \max }\left|\tau\left(z_{1}\right)-\tau\left(z_{2}\right)\right| \quad \forall\left(z_{1}, z_{2}\right) \in V_{j}^{2}:\left|z_{1}-z_{2}\right| \leq \delta .
\end{aligned}
$$

To conclude, we have established that, under the regularity assumption, for any $z$ in $\mathcal{O}$, there exist a neighborhood $V_{z}\left(=V_{j} \cap B_{z}(\delta)\right)$ and a real number $L_{z}$ such that we have

$$
\left|q_{0}\left(z_{1}\right)-q_{0}\left(z_{2}\right)\right| \leq L_{z}\left|\tau\left(z_{1}\right)-\tau\left(z_{2}\right)\right| \quad \forall\left(z_{1}, z_{2}\right) \in V_{z} .
$$

Proof of Proposition 2.1. By [10] (see Proposition 3) it is known that there exists a continuous $\gamma_{0}: \tau(\mathcal{A}) \rightarrow \mathbb{R}$ such that

$$
q_{0}(z)+\gamma_{0} \circ \tau(z)=0 \quad \forall z \in \mathcal{A} .
$$

We prove that if (12) holds and Assumption 2 is fulfilled, the function $\gamma_{0}$ is necessarily Lipschitz on $\tau(\mathcal{A})$. Once we have proved this, the fact that there exists a globally Lipschitz $\gamma: \mathbb{R}^{m} \rightarrow \mathbb{R}$ which agrees with $\gamma_{0}$ on $\tau(\mathcal{A})$, and thus which satisfies (8), follows by the Kirszbraun theorem (see, for instance, Theorem 2.10 .43 in [4]). To prove the fact that $\gamma_{0}: \tau(\mathcal{A}) \rightarrow \mathbb{R}$ is Lipschitz, we proceed by contradiction by supposing that, for all $n>0$, there exist two sequences $p_{n 1}$ and $p_{n 2} \in \tau(\mathcal{A})$ satisfying

$$
\left|\gamma_{0}\left(p_{n 1}\right)-\gamma_{0}\left(p_{n 2}\right)\right| \geq n\left|p_{n 1}-p_{n 2}\right| .
$$

Since $\tau(A)$ is compact, $\left\{p_{n 1}\right\}$ and $\left\{p_{n 2}\right\}$ have subsequences converging to some points $p_{1}^{\star}$ and $p_{2}^{\star}$ in $\tau(A)$. To simplify, we still denote by $\left\{p_{n 1}\right\}$ and $\left\{p_{n 2}\right\}$ these subsequences. Since $\gamma_{0}$ is continuous, the term $\left|\gamma_{0}\left(p_{n 1}\right)-\gamma_{0}\left(p_{n 2}\right)\right|$ is bounded, and hence $\left|p_{n 1}-p_{n 2}\right|$ tends to zero as $n$ tends to $\infty$. Thus, $p_{1}^{\star}=p_{2}^{\star}=p^{\star}$. Let $z^{\star} \in \mathcal{A}$ be any point of $\tau^{-1}\left(p^{\star}\right)$ and pick the set $Z \subset \mathcal{O}$ (see Assumption 2) as any subset of $V_{z^{\star}}$ (as defined in Lemma 2.2) having $z^{\star}$ in its interior. Let $U$ be the open set of $\mathbb{R}^{m}$, satisfying $U \cap \tau(\mathcal{O}) \subset \tau(Z)$, whose existence is secured by Assumption 2. We know that there exists $\bar{n}$ such that, for all $n>\bar{n}, p_{n 1}$ and $p_{n 2}$ are in $U \cap \tau(\mathcal{O})$. Hence, since $U \cap \tau(\mathcal{O}) \subset \tau(Z), p_{n 1}$ and $p_{n 2}$ are images of points $z_{n 1}$ and $z_{n 2}$ of $V_{z^{*}}$. Using (28) and (29), we have in this way shown the existence of sequences $\left\{z_{n 1}\right\}$ and $\left\{z_{n 2}\right\}$ of points of $V_{z^{\star}}$, satisfying $\left|q_{0}\left(z_{n 1}\right)-q_{0}\left(z_{n 2}\right)\right| \geq n\left|\tau\left(z_{n 1}\right)-\tau\left(z_{n 2}\right)\right|$, which, by virtue of Lemma 2.2, is a contradiction. This proves Proposition 2.1.

A drawback of Assumption 2 in Proposition 2.1 is that the function $\tau$ should be known in order to check whether it is open or not. In order to overcome this difficulty, in the next proposition we replace Assumption 2 with a more severe but checkable condition condition which refers to the following codistribution:

$$
\Omega_{e}\left(z_{1}, z_{2}\right):=\sum_{k=0}^{\infty} \operatorname{span}\left\{\frac{\partial}{\partial z} L_{f_{0}}^{k} q_{0}\left(z_{1}\right), \frac{\partial}{\partial z} L_{f_{0}}^{k} q_{0}\left(z_{2}\right)\right\} .
$$


Proposition 2.4. Let Assumption 1 hold. Assume, in addition, the existence of integers $c$ and $c_{e}$ such that (11) and

$$
\operatorname{dim} \Omega_{e}\left(z_{1}, z_{2}\right)=c_{e} \quad \forall\left(z_{1}, z_{2}\right) \in(\mathcal{O} \times \mathcal{O}) \backslash\left\{\left(z_{1}, z_{2}\right): z_{1}=z_{2}\right\}
$$

hold. Then there exists a globally Lipschitz function $\gamma(\cdot)$ satisfying (8).

Proof. We prove that there exists a $\rho>0$ such that for all $z_{1}, z_{2} \in \mathcal{A}$ the following holds:

$$
\left|q_{0}\left(z_{1}\right)-q_{0}\left(z_{2}\right)\right| \leq \rho\left|\tau\left(z_{1}\right)-\tau\left(z_{2}\right)\right| .
$$

From this, the existence of a globally Lipschitz $\gamma$ satisfying (8) follows from Proposition 3 of [10]. In order to prove (31), we proceed by contradiction by assuming that, for each integer $n$, we can find $z_{1 n}$ and $z_{2 n}$ in $\mathcal{A}$ satisfying

$$
\left|q_{0}\left(z_{1 n}\right)-q_{0}\left(z_{2 n}\right)\right| \geq n\left|\tau\left(z_{1 n}\right)-\tau\left(z_{2 n}\right)\right| .
$$

Since $\mathcal{A}$ is compact, there exist two subsequences of the sequences $\left\{z_{1 n}\right\}$ and $\left\{z_{2 n}\right\}$ converging to some points $z_{1}^{\star}$ and $z_{2}^{\star}$ of $\mathcal{A}$. For convenience we still denote by $\left\{z_{1 n}\right\}$ and $\left\{z_{2 n}\right\}$ these subsequences. Furthermore, since $q_{0}$ is bounded on $\mathcal{A}$, this implies that $\left|\tau\left(z_{1 n}\right)-\tau\left(z_{2 n}\right)\right|$ and therefore, with $(7),\left|q_{0}\left(z_{1 n}\right)-q_{0}\left(z_{2 n}\right)\right|$ tend to 0 as $n$ goes to $\infty$. Hence, $\left|\tau\left(z_{1}^{\star}\right)-\tau\left(z_{2}^{\star}\right)\right|=\left|q_{0}\left(z_{1}^{\star}\right)-q_{0}\left(z_{2}^{\star}\right)\right|=0$.

If $z_{1}^{\star}=z_{2}^{\star}=z^{\star}$, then, with Lemma 2.2, we know the existence of a neighborhood $V_{z^{\star}}$ and a real number $L_{z^{\star}}$ such that for each $n$ for which $z_{1 n}$ and $z_{2 n}$ are in $V_{z^{\star}}$, we have

$$
\left|q_{0}\left(z_{1 n}\right)-q_{0}\left(z_{2 n}\right)\right| \leq L_{z^{\star}}\left|\tau\left(z_{1 n}\right)-\tau\left(z_{2 n}\right)\right|,
$$

which contradicts (32).

We consider now the case $z_{1}^{\star} \neq z_{2}^{\star}$. Consider the extended system, with state $z_{e}=\left(z_{1}, z_{2}\right)$, defined as

$$
\dot{z}_{e}=f_{e}\left(z_{e}\right)=\left(f_{0}\left(z_{1}\right), f_{0}\left(z_{2}\right)\right), \quad q_{e}(z)=q_{0}\left(z_{1}\right)-q_{0}\left(z_{2}\right) .
$$

By Lemma 2.2 applied to this system (with $\Omega(z)$ and $\mathcal{O}$ replaced, respectively, by $\Omega_{e}\left(z_{1}, z_{2}\right)$ and $\left.\mathcal{O}_{e}=\mathcal{O} \times \mathcal{O} \backslash\left\{z_{e}: z_{1}=z_{2}\right\}\right)$, it turns out that there exist a neighborhood $V_{z_{e}^{\star}}$ of $z_{e}^{\star}:=\left(z_{1}^{\star}, z_{2}^{\star}\right)$ and $L_{z_{e}^{\star}}>0$ such that for all $z_{e}^{\prime}, z_{e}^{\prime \prime} \in V_{z_{e}^{\star}}$

$$
\left|q_{e}\left(z_{e}^{\prime}\right)-q_{e}\left(z_{e}^{\prime \prime}\right)\right| \leq L_{z_{e}^{\star}}\left|\tau_{e}\left(z_{e}^{\prime}\right)-\tau_{e}\left(z_{e}^{\prime \prime}\right)\right|,
$$

where $\tau_{e}\left(z_{e}\right)$, by linearity in $q_{0}$ of $\tau$ in (10), is given by $\tau_{e}\left(z_{e}\right)=\tau\left(z_{1}\right)-\tau\left(z_{2}\right)$. By taking $z_{e}^{\prime}=\left(z_{1 n}, z_{2 n}\right)$ and $z_{e}^{\prime \prime}=\left(z_{1}^{\star}, z_{2}^{\star}\right)$, it turns out that, for $n$ sufficiently large, $z_{e}^{\prime}$ and $z_{e}^{\prime \prime}$ belong to $V_{z_{e}^{\star}}$ and $z_{2 n} \neq z_{1 n}$. Hence, by using (33) and the definitions of $\tau_{e}(\cdot)$ and $q_{e}(\cdot)$, and the fact that $\left|\tau\left(z_{1}^{\star}\right)-\tau\left(z_{2}^{\star}\right)\right|=\left|q_{0}\left(z_{1}^{\star}\right)-q_{0}\left(z_{2}^{\star}\right)\right|=0$,

$$
\begin{aligned}
\left|q_{0}\left(z_{1 n}\right)-q_{0}\left(z_{2 n}\right)\right| & =\left|q_{0}\left(z_{1 n}\right)-q_{0}\left(z_{2 n}\right)-\left(q_{0}\left(z_{1}^{\star}\right)-q_{0}\left(z_{2}^{\star}\right)\right)\right|=\left|q_{e}\left(z_{e}^{\prime}\right)-q_{e}\left(z_{e}^{\prime \prime}\right)\right| \\
& \leq L_{z_{e}^{\star}}\left|\tau_{e}\left(z_{e}^{\prime}\right)-\tau_{e}\left(z_{e}^{\prime \prime}\right)\right|=L_{z_{e}^{\star}}\left|\tau\left(z_{1 n}\right)-\tau\left(z_{2 n}\right)-\left(\tau\left(z_{1}^{\star}\right)-\tau\left(z_{2}^{\star}\right)\right)\right| \\
& =L_{z_{e}^{\star}}\left|\tau\left(z_{1 n}\right)-\tau\left(z_{2 n}\right)\right|,
\end{aligned}
$$

which contradicts (32).

Remark 1. By going through the proofs of Propositions 2.1 and 2.4 and, in turn, of Lemma 2.2, it is possible to observe that the requirement of the existence of an 
open bounded set $\mathcal{O}$ which is backward invariant for $\dot{z}=f_{0}(z)$ (Assumption 1) is only motivated by the need of having the function $\tau(z)$ in (10) be well defined and $C^{2}$ (see the discussion before (16) in the proof of Lemma 2.2). In this respect the requirement in question can be replaced by the requirement that the function $\tau(z)$ in (10) be well defined and $C^{2}$ for all $z \in \mathcal{A}$. In this case, by inspection of the proof of the lemma (see in particular Claim 2 in the proof), it turns out that the regularity condition (11) must be replaced by

$$
\operatorname{dim} \Omega(z)=c \quad \forall z \in\left\{\phi_{f_{0}}(t, \varsigma) \quad: \quad t \leq 0, \varsigma \in \mathcal{A}\right\} .
$$

3. Conclusions. This paper complements the results of [10] by presenting a sufficient condition under which the output feedback stabilizer proposed in [10] can be taken to be locally Lipschitz. Under the technical conditions presented in Assumptions 1 and 2, it has been shown (see Proposition 2.1) that it suffices that the "observability" codistribution defined in (9) satisfies a constant rank property for concluding the existence of a locally Lipschitz stabilizer. The proposed result is expected to be useful in problems of output feedback stabilization and output regulation where regularity of the controller is an important issue.

Acknowledgment. The authors wish to thank A. R. Teel for having pointed out a gap in an earlier version of the manuscript.

\section{REFERENCES}

[1] V. Andrieu, Bouclage de sortie et observateur, Thèse de doctorat de l'École des Mines de Paris en Mathématiques et Automatique, Fontainebleau, France, 2005.

[2] V. Andrieu and L. Praly, On the existence of a Kazantzis-Kravaris/Luenberger observer, SIAM J. Control Optim., 45 (2006), pp. 432-456.

[3] F. Delli Priscoli, A. Isidori, And L. Marconi, Output regulation with redundant measurements, SICE J. Control Measurement System Integration, 1 (2008), pp. 92-101.

[4] H. Federer, Geometric Measure Theory, Springer-Verlag, New York, 1969 (reprint in 1996).

[5] J. P. Gauthier and I. Kupka, Deterministic Observation Theory and Applications, Cambridge University Press, Cambridge, UK, 2001.

[6] R. Hermann and A. J. Krener, Nonlinear controllability and observability, IEEE Trans. Automat. Control, 22 (1977), pp. 728-740.

[7] L. Marconi And L. Praly, Essential and redundant internal models in nonlinear output regulation, in Analysis and Design of Nonlinear Control Systems, A. Astolfi and L. Marconi, eds., Springer-Verlag, Berlin, 2007, pp. 125-146.

[8] L. Marconi and L. Praly, Uniform practical output regulation, IEEE Trans. Automat. Control, 53 (2008) pp. 1184-1202.

[9] L. Marconi, A. Isidori, ANd L. Praly, Robust asymptotic stabilization of nonlinear systems with non-hyperbolic zero dynamics, IEEE Trans. Automat. Control, to appear.

[10] L. Marconi, L. Praly, ANd A. Isidori, Output stabilization via nonlinear Luenberger observers, SIAM J. Control Optim., 45 (2007), pp. 2277-2298.

[11] L. Marconi And L. Praly, Some results on robust output feedback stabilization of nonlinear systems, in Proceedings of the 7th IFAC NOLCOS 2007, Pretoria, South Africa, 2007, pp. $551-556$. 